\title{
Implementation of Medical KIOSK: A Technical Review
}

\author{
Adithya R, Varunraj S and Sivaranjini. $\mathrm{A}^{1}$ \\ \{adithya191101@gmail.com, varunraj14522@gmail.com, asr.rae@psgtech.ac.in\} \\ Department of Robotics and Automation Engg., PSG College of Technology Coimbatore, India ${ }^{1}$
}

\begin{abstract}
Medical kiosks are an exquisite piece of ingenuity withinside the zone of personal healthcare. A kiosk is more than simply a computer with a touch screen housed in a box in today's world. Mechanical, computer hardware, software, peripherals, and embedded controllers are all integrated within it. Its construction necessitates high-level subject understanding and mental fortitude. The hospital Kiosk project aims to pave the way for more complex hospital service applications with user interfaces in the future. The purpose of this research article is to look at some of the ways that have been utilized to develop a medical kiosk and identify and investigate some of the issues that have been raised and potential solutions provided by academics.
\end{abstract}

Keywords: KIOSK, phonocardiograms, electrocardiograms.

\section{Introduction}

The state of complete mental, physical, and social well-being is defined as health. A person's health impacts him and others around him and the entire society in a variety of ways, both directly and indirectly. Maintaining excellent health has never been more essential since it has a beneficial impact on nearly every area of our lives. It helps you live longer and feel better about yourself, and clinical studies have shown that those who follow a healthy lifestyle have reduced stress and anxiety levels. A proper diet and a healthy diet help safeguard your eyesight. Maintaining your health leads to an increase in your self-assurance.

Healthcare services are offered to assist people in keeping track of their health status, taking steps to address detected concerns, and avoiding severe diseases. People should receive constant and comprehensive care from health care centers. It also aids in concerning the patient with public health well-being services and various communal welfare activities provided by the related governing bodies and other administrations. A health care center's other important function is to provide high-quality health and social services to the underprivileged. People receive the initial set of professional care from health care centers, which incorporates a proactive strategy that includes many preventative measures, self-care, and chronic illness management. 


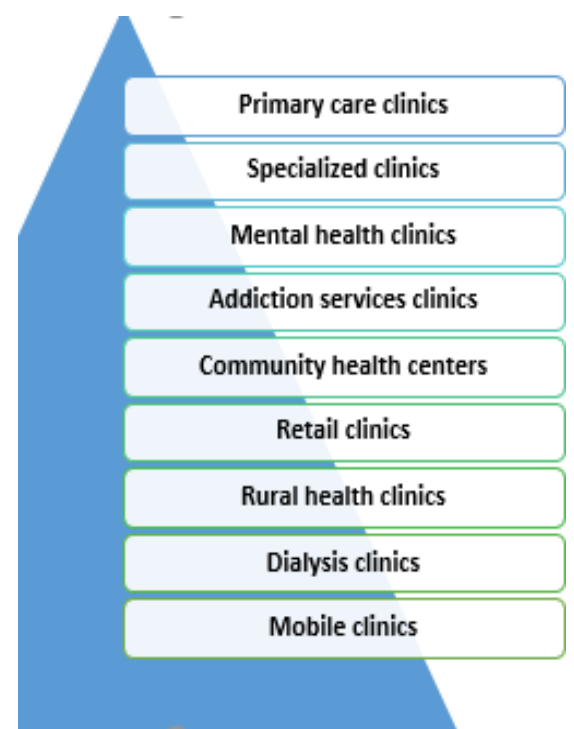

Fig. 1. Types of health care clinics

The healthcare industry also plays an essential role in addressing the demand for people health. The collective well-being of community members is referred to as community health. These communities share similar health individualities, societies, and economic circumstances and live in the same geographical territory. In communities where socioeconomic circumstances impede health equity, health care facilities frequently function as the primary care provider [1].

Healthcare providers can now deliver improved services because of automation. Improved efficiency, better patient care, and retrieval of patient data are just a few of the benefits. Healthcare automation also enables health providers to keep a close eye on patients and remind them of follow-up appointments.

A medical kiosk eliminates the need for doctor or medical personnel to administer medical tests. Medical kiosks enable self-service methods for obtaining medical attention, such as preliminary medical tests and obtaining information on specific health concerns.

\section{Challenges encountered while implementing a kiosk system:}

Even in the current scientific period, providing quality healthcare to rural and isolated places has been difficult. According to several studies, people in rural regions have less access to healthcare information than people in metropolitan areas. Several public awareness campaigns were undertaken to enlighten rural residents about the importance of good health. Regional medical camps were also set up to address the problems, but neither was consistent [2].

- Accessibility:

Since one of the primary objectives of a medical kiosk is to benefit underprivileged people, particularly those who reside in rural or remote areas, literacy is an important aspect to 
consider while developing the kiosk's system. Also, with more people living aboard, tourists traveling to international destinations immigrants settling in new countries, and, our multilingual world needs to cater to different languages. Unfamiliarity with the language of the device is the lack of information about electronic and digital devices and how to use the device, users and healthcare providers ignorance with types of services. Users are not familiar with kiosks' benefits and their services.

\section{- Factors affecting the accuracy of the sensors:}

Multiple distinct sensors are utilized to assess various health indicators of the body, and these sensors are interfaced with a processing computer for further computation of findings. Given that the results of all of these sensors' measurements will be used by medical specialists to provide advice on a person's health, there is a demand for accuracy. There are a variety of factors that impact the sensors' performance efficiency and accuracy.

The placement and arrangement of the sensors in the machine's structure have a significant impact on the accuracy of the measurements. For example, to acquire an accurate result, the body temperature should be measured by placing the temperature sensor at a nominal distance from the forehead; similarly, each sensor should be set at a specific position and orientation for optimal accuracy.

Varying types of kiosks allow for different placements of sensors that are used for measurements, which necessitates the user changing their posture to fit the sensor positioning while measuring. As a result, there is a deviation from accuracy. Different postures, for example, can produce a 20 percent difference in recorded systolic/diastolic blood pressure (MacWilliam, 1933).

When you go to a medical facility for a checkup, the physicians make sure you're relaxed by asking you to take a few deep breaths before they examine your body. Studies have proven that the accuracy of the sensors can also be influenced by the user's physiological and mental condition.

This can trigger a difference of more than $20 \%$ in cardiovascular measurements.

If sufficient maintenance is not provided for any machinery, it will wear down with time, and the same is applicable for medical kiosks. Since the kiosk uses delicate medical equipment, it is vital to perform ongoing maintenance in order to obtain correct results.

\section{- Problems faced at data level:}

The Kiosk consists of various sensors that operate on multiple parameters, each with its own data format and communication protocol for sending and receiving data. For example, most temperature sensing devices communicate using the Universal serial bus protocol, whereas an Upper- Hand Blood Pressure Monitor communicates via serial port, making it difficult to accept instructions from and transmit output to the CPU.

In order to keep track of the medical records of the people who use the kiosk on a regular basis, a unique ID for each person must be created, which includes personal information (name, address, contact details, medical history, etc.) that should not be editable or viewable by anyone other than the owner of the information. As a result, personal information confidentiality must be considered. Since the measurements recorded by the devices represent one's current health status, which also falls under personal information, effective means of privacy protection play a key role. 
Typically, when a health checkup is performed at a physical clinic, the report is analyzed by a medical professional in order to provide accurate information about one's health condition and, if necessary, to provide medication. As a result, the kiosk should provide a means for measured data collected from a sensor to be compiled in a readable format that can be overlooked by a healthcare professional.

\section{- Other problems:}

When it comes to medical testing, hygiene is crucial. Several kiosks require a small amount of blood $(2 \mathrm{ml})$ from the user in order to measure the oxygen saturation level in the blood, which can lead to fatal infections if performed incorrectly. This also detracts from the kiosk's purpose and raises concerns about its usability.

Because the sensors used in medical kiosks are electrically powered digital sensors with high sensitivity, when they are used continuously for an extended period of time without a break, the software and hardware struggle to keep up, causing the device to produce less reliable data and, in the worst-case scenario, software crashes.

Almost all types of kiosks have one or more digital screens, most of which are touchsensitive, that act as a human-machine interface (HMI) to provide instructions and guide the user step by step through the process. These screens are also used for logging and view almost any visual information related to the kiosk, including personal data, so it is important to consider the user's privacy when designing the display system.

\section{Clinical Kiosks}

Here is a limited amount of evidence-based research on kiosk implementations or outcomes regarding process efficiency, cost savings, or clinical care and management.

Self-service kiosks have been employed in a variety of businesses outside of healthcare to expand service offerings, boost throughput, and generate new revenue. To aid corporate strategy planning and to inform large-scale healthcare implementations, qualitative and quantitative data are required. The healthcare business, on the other hand, has been slow to adopt kiosk technology. There is a significant gap in the literature, and any generalizability in healthcare is limited. Check-in kiosk technology has been reported to improve operational efficiency and clinic throughput in various cases. Implementation of medical Kiosks can be used to track the evolution of chronic illnesses, rate clinical outcomes, assist with diagnosis, and triage patients, for example.

\section{A. What's in a health care kiosk?}

The sensors (input devices) and output devices in a healthcare kiosk are diverse. Input devices for interactive computer kiosks include touchscreens, trackballs, computer keyboards, and pushbuttons. Commercially, touchscreen kiosks are utilized as industrial appliances to reduce lines, eliminate paper, and improve efficiency and service.

The following main blocks make up the fundamental sensors used in the kiosk system's architecture: 


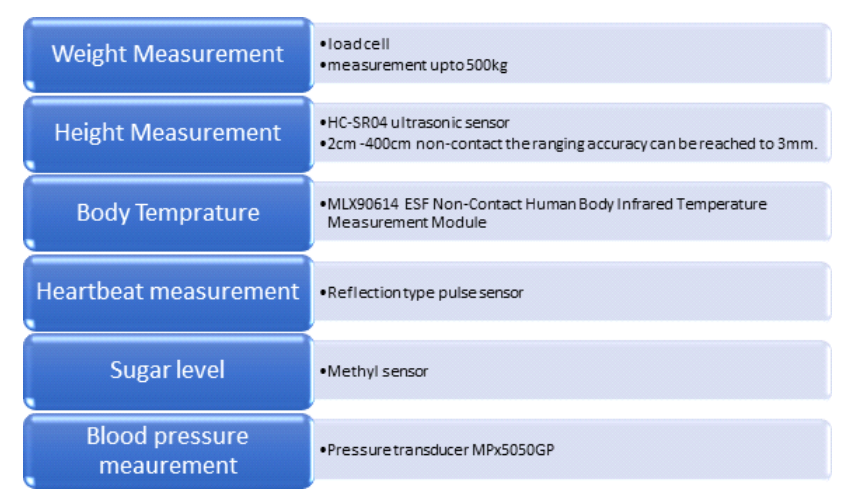

Fig. 2. Sensors implemented in a medical kiosk

\section{- Weight measurement:}

Weight is measured using load cells. A load cell is a transducer or sensor that translates an applied force into an electrical signal. This electrical signal might be a voltage change, current change, or frequency shift dependent on the load cell and circuitry employed. Single Point Load Cells are used to process various applications that require weight control platforms on a tiny scale. This load cell design provides many advantages over others, including high accuracy, minimal profile, and the ability to compensate for off-center loading. It also has a small size and great accuracy. The maximum measurement of the load cell is $500 \mathrm{Kg}$.

\section{- Height measurement:}

An ultrasonic sensor is a gadget that detects objects using ultrasonic sound waves for measuring the distance to an item. An ultrasonic sensor has a transducer that emits and receives ultrasonic pulses to communicate information about the proximity of an object. Highfrequency sound waves reverberate off of surfaces, creating unique echo patterns. The HCSR04 ultrasonic sensor is used to determine a person's height. It is powered by a $5 \mathrm{~V}$ DC source with a standby current of less than $2 \mathrm{~mA}$. It provides excellent precision and reliable readings in a simple-to-use design.

\section{- Blood pressure measurement:}

Measure the blood pressure regularly to aid your doctor in detecting any health issues early. Many people are unaware that they have high blood pressure since there are no warning signs or symptoms. The pressure sensor's output is divided into two signals. The serial ADC receives both signals. Because the MPX5050GP pressure sensor outputs between $0.2 \mathrm{~V}$ and $4.7 \mathrm{~V}$, the initial signal is sent straight into the microcontroller without being amplified. The oscillation signal and the cuff pressure signal are included in this signal. The second signal is 256 inputs via a two-pole high pass filter to suppress the cuff pressure signal and increase oscillation.

\section{- Body temperature:}


The sound of a person's heartbeat is the sound of the heart valves contracting or expanding as they push blood from one region to another. The heartbeat rate is the number of heart beats per minute, and the pulse is the heartbeat, which may be felt in any artery near the skin. There are several ways for measuring heart rates, including phonocardiograms (PCG), electrocardiograms (ECG), blood pressure waveforms, and pulse meters, but these procedures are clinical and costly.

The heartbeat sensor works based on photoplethysmography. It monitors the change in blood volume via any body organ that results in a change in light intensity through that organ (avascular region). The timing of the pulses is particularly essential in situations where the heart rate is to be monitored. The pace of heartbeats determines the flow of blood volume, and because the blood absorbs the light, signal pulses are equal to heartbeat pulses. Photoplethysmography is classified into two kinds.

One method is the Transmission kind. Light released from the light-emitting device is transmitted through any vascular part of the body, such as the earlobe, and is detected by the detector; another kind is the regions that reflect the Reflection type, light emitted from the light-emitting device. Reflection-type pulse sensors emit infrared, red, or green light (550nm) towards the body and use a photodiode or phototransistor to detect the quantity of light reflected. Because oxygenated hemoglobin in the blood of the arteries absorbs incident light, we may detect the pulse wave signal by measuring the blood flow rate (change in blood vessel volume) that varies over time as a result of cardiac contractions.

- Sugar level:

Measuring sugar levels is used as a screening technique for diabetes mellitus (diabetes), and it is also an essential tool in evaluating the ill patient, particularly in the young and old. Blood glucose extremes that are potentially life-threatening can be recognized, allowing the patient, caregiver, or health professional to respond to high (hyperglycemia) and low (hypoglycemia) blood glucose levels by changing the diet or administering insulin. In a disposable 'test strip,' a drop of blood is applied which is then placed into an electronic blood glucose meter, and the level of glucose in the blood is measured. The meter detects and displays in units of $\mathrm{mg} / \mathrm{dL}$ or $\mathrm{mmol} / \mathrm{L}$ the response between the test strip and the blood.

- SpO2 Measurement:

Pulse oximetry is a simple, low-cost, non-invasive method for monitoring oxygenation. It measures the proportion of oxygen-saturated hemoglobin. Oxygen saturation should always be greater than $95 \%$. However, it may be lower in patients with long-standing respiratory illness or cyanotic congenital heart disease, depending on disease severity. Oximeters operate on the principles of spectrophotometry: the systolic component of the absorption waveform's relative absorption of red (absorbed by deoxygenated blood) and infrared (absorbed by oxygenated blood) light corresponds to arterial blood oxygen saturation. Measurements of relative light absorption are taken several times per second, and the system processes them to provide a new value every $0.5-1$ second that averages the results from the previous three seconds.

Through 5-10 $\mathrm{mm}$ of tissue, two light-emitting diodes, one red and one infrared, are positioned opposite to their respective detectors. Probes are often placed on the fingertip. However, the earlobes and forehead are occasionally used as substitutes. According to one study, the ear lobe is not a viable location for measuring oxygen saturation. Recent research shows that the earlobe is used in patients admitted to intensive care units for coronary artery bypass surgery. Sensors of the 'wrap' or 'clip' variety are commonly used in probes. 


\section{Methodologies}

Below mentioned are some of the methods used by scholars to overcome the problems faced while implementing a kiosk.

\section{- Compatibility with users:}

Because the kiosk makes use of several professional biological devices, users may want instruction when using it for the first time. It is quite simple to understand how to use for typical folks with only a high school education [3]. Professional support is recommended for the use of elderly individuals.

In order for the kiosk system to be easily accessible and approachable without hesitation, it must be suitable for the needs of all people when it comes to machine-user interaction. When developing the kiosk's user interaction system, a number of factors are taken into account. Seeing as people will be receiving instructions and information that are critical for the machine's efficient operation, the system should include both hardware and software features to display the instructions and information on the LCD screen, as well as through a speaker for users who may have a significant level of visual impairment. The system also disseminates various instructional messages in the form of media and other formats.

\section{- Multilingual operation:}

The medical kiosk system is designed to work with all of the major languages spoken across the world, as well as some of the local languages used in the area where it will be installed. Because the people will be receiving instructions and information that are critical to the machine's efficient operation, all of the data that will be displayed and the audio output from the speakers should be provided in a variety of languages from which the user can select their preferred language.

\section{- $\quad$ Privacy of the user:}

Considering medical kiosks are to be installed for the general public in an easily accessible location, such as the setting of an ATM, private space is not assured while a single person uses it. To overcome this issue, privacy walls for the LCD panel where the information is to be displayed should be supported by the kiosk's structure. According to many surveys, the size of the screen has a significant impact on the user's feeling of privacy while using a kiosk; people are more comfortable with a 12 inch or 15 -inch screen with privacy dividers than with a 17-inch screen with a privacy partition.

- Data Transfer:

All of the sensor data is to be routed into a central computer for further processing. For interaction between the sensor and the processor, each sensor in the kiosk utilizes a distinct output data format and communication protocol; this becomes a complex job for the software to manage, and the software inevitably malfunctions. To address this issue, Guiqin Sun and his colleagues [1] established a method for making the entire integration work by establishing a single data format for all sensors.

By assigning distinct values to a set of multiple bytes of data, these values point to different information such as the message header, data length, module number, command, response, and the remaining data body. For the various pieces of information listed above, unique integer codes are assigned and utilized as labels beneath each group of bytes. 
G. Data privacy:

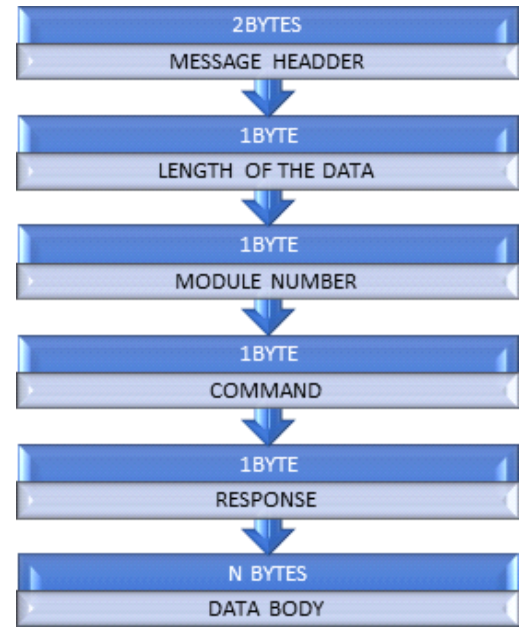

Fig. 4. KIOSK Architecture

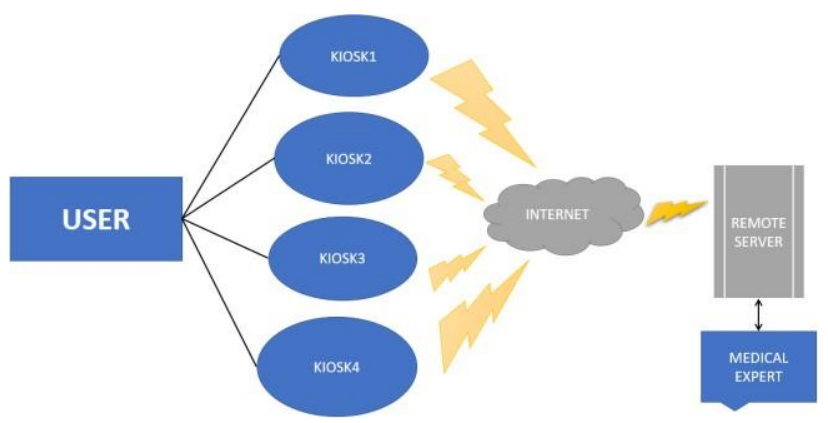

Fig. 3. Common Data Format

\section{- Hygiene:}

When it comes to biological testing, the first thing to consider is the user's safety; all sensors should be constructed in such a way that they do not harm the user in any way. Because the same sensor will be used to test several persons, it is vital to verify that the sensors do not pass on infections from other people. The majority of the testing is done with non-contact sensors. Sensors that require contact are only used if they are touch contact sensors. A sugar level sensor, for example, requires a drop of blood on a prepared medical strip to measure blood glucose levels, which necessitates medical supervision, making it unsuitable for use in a kiosk.

- Upload to server: 
Doctors will analyze the sensor data and provide feedback on the report, as well as prescribe medication depending on their current and past health. In order to complete the task, communication between the machine and the doctors had to be established. To perform this objective, a group of researchers devised a method for backing up all obtained data to a distant server, which can be accessed via a web interface to display user details. The information includes the most recent measurement data obtained from the machine, as well as the results of all prior tests performed on the equipment by the user.

Personal information and medical records are among the data received from users for registration, and they should be kept private and safe from other users, readable only by the authorized user and other authorized personnel, and editable only by the user. The data will also include the kiosk's current and previous test results. In light of this, data must be securely encrypted while being stored locally and while being uploaded to the server.

Guiqin Sun and his colleagues [1] devised a solution for this problem by immediately uploading the compiled test results to a remote server via a 128-bit encrypted HTTPS protocol, ensuring that the data is safe on the internet's data path. When there are network issues, the data is always encrypted before archiving, and it is automatically deleted if the network is still down and when the data backup is completed.

A reputable third-party provider can provide the service for securely accessing this data. As stated in [2,], the data can be safely backed up to Microsoft Azure (a cloud computing service provider) on which the portal will be created and built, which will run on a Microsoft SQL SERVER EXPRESS 2016 in the Azure cloud. C\# is used to develop all of the admin services. The overall portal and analytical portal are encrypted using a Dynamo DB.

\section{H. Accuracy of the sensors:}

Because the location and orientation of the sensors can affect the test results, the structural architecture must be designed in such a way that it accommodates the sensors in a nominal position and orientation to support each user.

As suggested in [9] there are two methods to handle this problem: one is to create a design that adjusts to diverse users, and the other is to employ (public) data on anthropometry, biomechanics, or ergonomics at the population level. An automatic device adaptation for any specific user might be realized using the population-fitting functions of the optimal device design.

The study [9] presents an approach for developing and optimizing a self-service physiological kiosk. Few design studies in this domain give a formalization of the problem, methods for optimizing it, and demonstration utilizing a broad, appealing design problem. The usage of a specialized design model and associated design and optimization approach helps to ensure that the implementation is accurate. In ubiquity, precise physiological measurements are required.

To provide multiple solutions, the problems are deconstructed, codified, and targeted. As a result of this approach, measurement noise is reduced, and an optimal fit in terms of sensor placement is discovered.

\section{Discussions}

In this study, we discovered a variety of issues that arise while implementing a healthcare kiosk and solutions to most of those problems. However, even though we found solutions for 
most issues, each one has its own set of benefits and drawbacks when dealing with actual people.

The Low-Cost Community Healthcare Kiosk [1] was not assured in the initial trials. Thus, actual community trials were needed, as well as more testing results to be collected and evaluated. The system's usability and robustness can be improved further based on them. One of the most critical aspects of the Low-Cost Community Healthcare Kiosk [1] is that it addressed the issue of user data privacy and developed data encryption methods.

However, there is still a need for a medical professional to evaluate the collected data to provide advice and medical prescriptions. In the future, a medical kiosk may integrate an AI algorithm that can perform the function of a medical expert. In future advances, including AI into the kiosk system could help build a far better overall user experience. Future research should concentrate on connecting stand- alone kiosks to the Internet and healthcare, a system that also allows patients to view portions of their information remotely.

In an emergency, kiosks are demonstrated to be more effective than nurses or physicians in gathering patient medication information, improving documentation and identifying risk factors, and increasing patient compliance with clinical guidelines and instructions. The kiosks' design disclosed that the hardware was reasonably inexpensive and that the system was more cost-effective than booklets to increase the number of people who received nutritional information.

Although some specifics of the hardware and development costs were covered, we did not have a complete cost analysis of the kiosk design, development, deployment, and ongoing maintenance. Development cost analysis, as well as software and hardware management, must be addressed.

\section{References}

[1] Guiqin Sun, Shenyi Tao, Yongqiang Lu, Yu Chen, Yuanchun Shi, Ni Rong, Rui Wang, Xiaojuan Lu "A Low-Cost Community Healthcare Kiosk" 2011 IEEE 13th International Conference on eHealth Networking, Applications and Services.

[2] N. Komal Kumar, Dr. R. Bhavani, Dr. A. Mohan, D. Vigneswari "ISSK-An Integrated Self Service Kiosk for Health Monitoring and Management" INTERNATIONAL JOURNAL OF SCIENTIFIC \& TECHNOLOGY RESEARCH VOLUME 8, ISSUE 09, SEPTEMBER 2019.

[3] Yu-Feng Yvonne Chan, Roxanne Nagurka, Suzanne Bentley Edgardo Ordonezand, William Sproule, "Medical Utilization of Kiosks in the Delivery of Patient Education: A Systematic Review".

[4] Kadam Pooja Laxman Lilavati, Mishra Preeti Ravindra Vimla "Implementation of Information Kiosk for BARC Hospital," Project Report.

[5] Sanjana S, Mr.Rathnakara S, "DESIGN OF INTERFACE BOARD FOR MEDICAL KIOSK BASED ON "OFF-THE-SHELF" PLATFORM," International Research Journal of Engineering and Technology(IRJET), Volume: 04 Issue: 07| June-2017.

[6] Blake Lesselroth, MD, MBI1,2,*, William Eisenhauer, MEng1, Shawn Adams1,2,David A. Dorr, MD, MS2, Christine Randall1, Paulette Channon1,Kas Adams, MPH1, Victoria Church, RN, CNS1, Robert Felder, DDS, MPH1,David Douglas, MD, "Simulation Modeling of a Check-inand Medication Reconciliation AmbulatoryClinic KioskJournal of Healthcare Engineering. Vol. 2 - No. 2. 2011 Page 197-222.

[7] Harshada R.Nawale, Gayatri S. Nikumbh, Rutuja J. Sonawane, Arvind P. Meshram, "HEALTHCARE KIOSK USING IOT", nternational Journal of Computer Engineering and Applications, Volume XII, Special Issue, April18, www.ijcea.com ISSN 2321-3469.

[8] Riyanto Sigit, Zainal Arief, Mochamad Mobed Bachtiar, "Development of Healthcare Kiosk for Checking Heart Health", EMITTER International Journal of Engineering Technology Vol.3, 
[9] No.2, December 2015

[10] Lyu, Y., Vincent, C. J., Chen, Y., Shi, Y., Tang, Y., Wang, W., Liu, W., Zhang, S.,Fan, K., \& Ding, J. (2015). "Designing and optimizing a healthcare kiosk for the community", Applied Ergonomics, 47,157-169. 